\title{
Advantage of using deep inspiration breath hold with active breathing control and image-guided radiation therapy for patients treated with lung cancers
}

\author{
KR Muralidhar', Rajib Lochan Sha², Birendra Kumar Rout ${ }^{3}$, P Narayana Murthy ${ }^{4}$ \\ ${ }^{I}$ Department of Radiation Physics, American Oncology Institute, Hyderabad, India \\ ${ }^{2}$ Radiological Safety Division, Atomic Energy Regulatory Board, Mumbai- 94, India \\ ${ }^{3}$ Department of Medical Physics, Aditya Birla Hospital, Pune, India \\ ${ }^{4}$ Department of Physics, Nagarjuna University, Guntur, India
}

Received October 26, 2014; Revised December 05, 2014; Accepted January 15, 2015; Published Online January 22, 2015

\section{Original Article}

\begin{abstract}
Purpose: The aim of this study was to evaluate the irradiated volume and doses to the target, heart, left lung, right lung and spinal cord, the number of segments and treatment time by using moderated deep inspiration breath hold (mDIBH) with active breathing control (ABC) and image-guided radiotherapy (IGRT) for patients treated with lung cancers. Methods: The suitability of this technique for lung patient treated with $A B C$ was investigated and the solutions to achieve better treatments were discussed. Eleven lung cancer patients (3 left-sided and 8 right sided lesions) with stages I-III underwent standard free breath (FB) and $\mathrm{ABC}$ computed tomography (CT) scans in the treatment supine position. This can be achieved by applying respiratory manoeuvres, such as $\mathrm{mDIBH}$, during which the threshold volume utilized is defined as $75-80 \%$ of the maximum aspiratory capacity. Five to seven, 6-MV photon beams with optimized gantry angles were designed according to the tumor location to conform to the PTV while sparing as much heart, spinal cord, and contra lateral lung as possible. For eleven patients, treatment planning using mDIBH CT data with intensity modulated radiation therapy (IMRT) was then reoptimized on the free breathing data set for comparison. The studied parameters of the plans for each patient were evaluated based on the average of the minimum, mean, and maximum difference in dose, the range of difference, and the $p$-value using two-tailed paired $t$ test assuming equal variance. Results: The average volume of the planning target volume (PTV) in 11 patients increased to $1.32 \%$ in ABC compared to FB. The average volume of heart in 11 patients decreased to $2.9 \%$ in ABC compared to free breathing IMRT. In the case of lungs, the volume increased to $27.5 \%$ and $25.85 \%$ for left and right lungs, respectively. The range of mean difference in dose to the PTV in 11 patients was $-54 \mathrm{cGy}$ to $230 \mathrm{cGy}$ with $\mathrm{ABC}$ technique when compared with free breathing. The range of mean dose difference of heart in 11 patients observed were -88 cGy to $66 \mathrm{cGy}(p<0.0410)$ between ABC and FB. The range of maximum dose difference to the spinal cord in 11 patients were -1592 cGy to $190 \mathrm{cGy}(p<0.041)$ with $\mathrm{ABC}$ technique when compared with FB IMRT. Monitor units (MUs) were -22.9\% less in ABC compared to FB. Segments were more in ABC compared to FB for about $16.39 \%$ on an average. The average of minimum, mean and maximum difference in dose to the right lung and left lung were less in $\mathrm{ABC}$ compared with FB. Conclusion: In most of the cases, IGRT with ABC significantly reduces the mean dose to heart, right lung, left lung, and spinal cord compared with FB. Discrepancy observed in few cases made the statistical data inconsistent. Depending on anatomy and arbitrary phase of the breathing cycle, the results may vary and for better outcome of the results optimum treatment procedures need to follow.
\end{abstract}

Keywords: Active breath control; mDIBH; Lung tumors

\section{Introduction}

Treatment modalities for the lung cancers in radiation oncology field are rapidly evolving. More than $60 \%$ of patients with lung cancers undergoing radiation therapy (RT) during the course of their disease, $45 \%$ for initial treatment and $17 \%$ for palliation. ${ }^{1-2}$ Understanding the normal anatomy is very important for delineating the tumour. Abnormal and doubt- ful structures should be evaluated for fluid content to exclude pericardial fluid in a recess. ${ }^{3-4}$ For better delineating the target and normal structures computed tomography (CT), magnetic resonance imaging (MRI) and positron emission tomography (PET) were used together. It is also now possible to do 2D-2D match and 3D-3D match with the help of electronic portal 
imaging device (EPID) and cone beam CT (X-Ray volume imaging (XVI)) respectively to measure and account for interfraction variations. In motion management intrafraction variation is also one of the important factors to account.

Intrafraction motion is caused by (a) respiratory (b) skeletal-muscular, (c) cardiac, and (d) gastrointestinal systems. The problems of respiratory motion during radiotherapy are image-acquisition limitations, treatment-planning limitations and radiation-delivery limitations. Methods to account for respiratory motion in radiotherapy ${ }^{5}$ are

- motion-encompassing methods,

- respiratory gating methods,

- breath-hold methods,

- forced shallow breathing with abdominal compression, and

- real-time tumor-tracking methods.

In our study active breathing control ( $A B C$ ) was used which is under breath-hold method. The purpose of this study is to investigate the impact of using $A B C$ treatment with moderated deep inspiration breath hold $(\mathrm{mDIBH})$ on the PTV, lungs, heart, and spinal cord. Dose to the PTV and critical structures, volume of organs, treatment time, number of segments and monitor units were compared in free breath (FB) to $\mathrm{ABC}$. This procedure helps us to check the suitability of $\mathrm{ABC}$ technique to any lung patient. In case of contrary results the solutions to achieve the better results are also given.

\section{Methods and Materials}

One of the main benefits of the active breathing coordinator 6-8 is that it is useful for tumor immobilization. Eleven lung cancer patients (3 left-sided and 8 right-sided lesions) with Stages I-III underwent standard FB and ABC CT scans in the treatment position. Among eleven patients, four are female and seven are male with age from 30 to 82 years. Sixteen slice Siemens Somatom CT scanner was used to scan $2 \mathrm{~mm}$ slices at the predefined threshold level. This can be achieved by applying respiratory manoeuvres, such as $\mathrm{mDIBH}$, during which the threshold volume utilized is defined as $75-80 \%$ of the maximum aspiratory capacity. Applying mDIBH technique increases the total volume of the lungs, moving the chest wall anterior and the diaphragm inferior. For patient set-up, we used all-in-one (AIO) (Orfit, Jericho, New York, USA) immobilization system and Vac-Lok hemibody cast (MED-TEC, Orange City, IA, USA). Patients with immobilization and $\mathrm{ABC}$ underwent $\mathrm{CT}$ simulation. Images with moderate mDIBH from CT were sent to three-dimensional (3D) $\mathrm{XiO}$ treatment planning system (TPS) (CMS Inc., St. Louis, MO, USA) through DICOM 3.0 Network.

The clinical target volume (CTV) and planning target volume (PTV) were derived by applying $8 \mathrm{~mm}$ and $7 \mathrm{~mm}$ margin to gross tumor volume (GTV) and CTV, respectively.
Additional $5 \mathrm{~mm}$ margin was outlined to include the shift in tumor position during ABC gating. ${ }^{9-10}$ Fused magnetic resonance imaging (MRI) and position emission tomography (PET) CT images were used for delineation of the target structures. Simultaneously, contouring was done on FB images also. Five to seven 6-MV photon beams with suitable gantry angles were designed ${ }^{11-13}$ (Figure 1) according to the tumor location to conform to the PTV while sparing as much heart, spinal cord, and contra lateral lung as possible. Additionally, transverse CT slices and digitally reconstructed radiographs (DRR) from the $\mathrm{BEV}$ were used to optimize field placement. Planning for $5000 \mathrm{cGy}$ in 10 fractions was done using step and shoot IMRT technique (Elekta Synergy linear accelerator) with $1 \mathrm{~cm}$ segment size and maximum intensity levels of 10 . The segmented beam data was sent to the machine console for treatment.

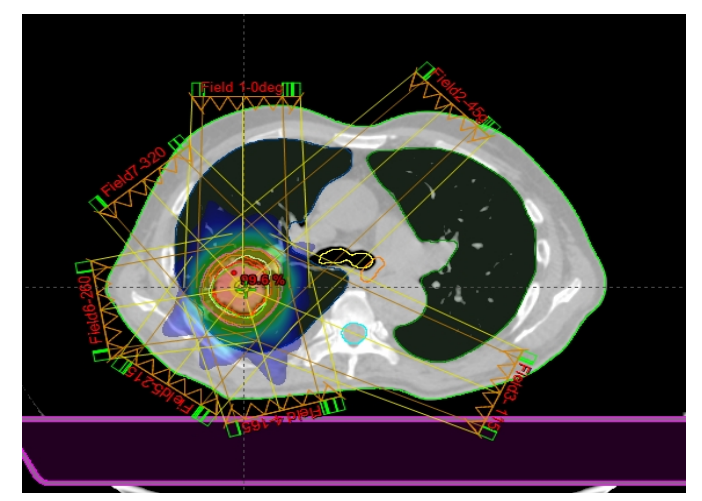

FIG. 1: Beam placement in lung cases in sagittal transverse view.

For eleven patients, treatment planning using mDIBH CT data with IMRT was then reoptimized on the free breathing data set for comparison with the same plan that was used for ABC. Dose-volume histograms for the PTV, heart, right lung, left lung, and spinal cord were analyzed. The studied parameters of the different plans for each patient were evaluated based on the minimum, mean and maximum difference, the range of difference, and the $p$ value using two-tailed paired $t$ test assuming equal variance. ${ }^{14}$ The change in volumes of PTV, lungs, heart and spinal cord were recorded. In addition, the PTV volume receiving at least $95 \%$ of the prescription dose $\left(\mathrm{V}_{95}\right)$ and $105 \%$ of the prescription dose $\left(\mathrm{V}_{105}\right)$, ipsilateral and contra lateral lungs volume receiving at least $20 \mathrm{~Gy}\left(\mathrm{~V}_{20}\right)$, heart volume receiving more than $30 \mathrm{~Gy}\left(\mathrm{~V}_{30}\right)$, spinal cord maximum dose and absolute volume receiving more than 45 Gy $\left(\mathrm{V}_{45}\right)$, the number of monitor units (MUs) and segments were compared between FB and ABC for a single fraction of 5 Gy.

\section{Results}

\section{Analysis of volume and dose in $\mathrm{ABC}$ and $\mathrm{FB}$ PTV}

The average volume of the PTV in 11 patients increased to $1.32 \%$ in $\mathrm{ABC}$ compared to $\mathrm{FB}$ with the range of difference is 
from $-33.9 \%$ to $22.29 \%(p<0.2536)$ as shown in Figure 2 . Maximum difference and minimum differences were $-33.9 \%$ $(-19.13 \mathrm{cc})$ and $-0.70 \%(-2.3 \mathrm{cc})$, respectively. The range of mean difference in dose to the PTV in 11 patients was -54 cGy to $230 \mathrm{cGy}(p<0.5447)$ with $\mathrm{ABC}$ technique when compared with FB IMRT. The average of minimum, mean and maximum differences in dose to the PTV were -222.45 cGy $(p<0.2889), 14.09$ cGy $(p<0.5447)$ and 33.6 cGy $(p<$ 0.5268 ) respectively. The range of minimum difference in dose was from -2002 cGy to 407 cGy and the range of maximum difference in dose was from $-280 \mathrm{cGy}$ to $430 \mathrm{cGy}$. The minimum, maximum and mean dose to the PTV in both FB and ABC are shown in Table 1 . The $\mathrm{V}_{95}$ and $\mathrm{V}_{105}$ of the PTV were analyzed in both $\mathrm{ABC}$ and $\mathrm{FB}$ techniques as shown in Figure 3. The mean difference of PTV volume receiving $95 \%$ and $105 \%$ of dose are from $0 \%$ to $-10 \%(p<0.00815)$ and $26 \%$ cc to $-82 \%$ cc $(p<0.211)$ respectively.

\section{Heart}

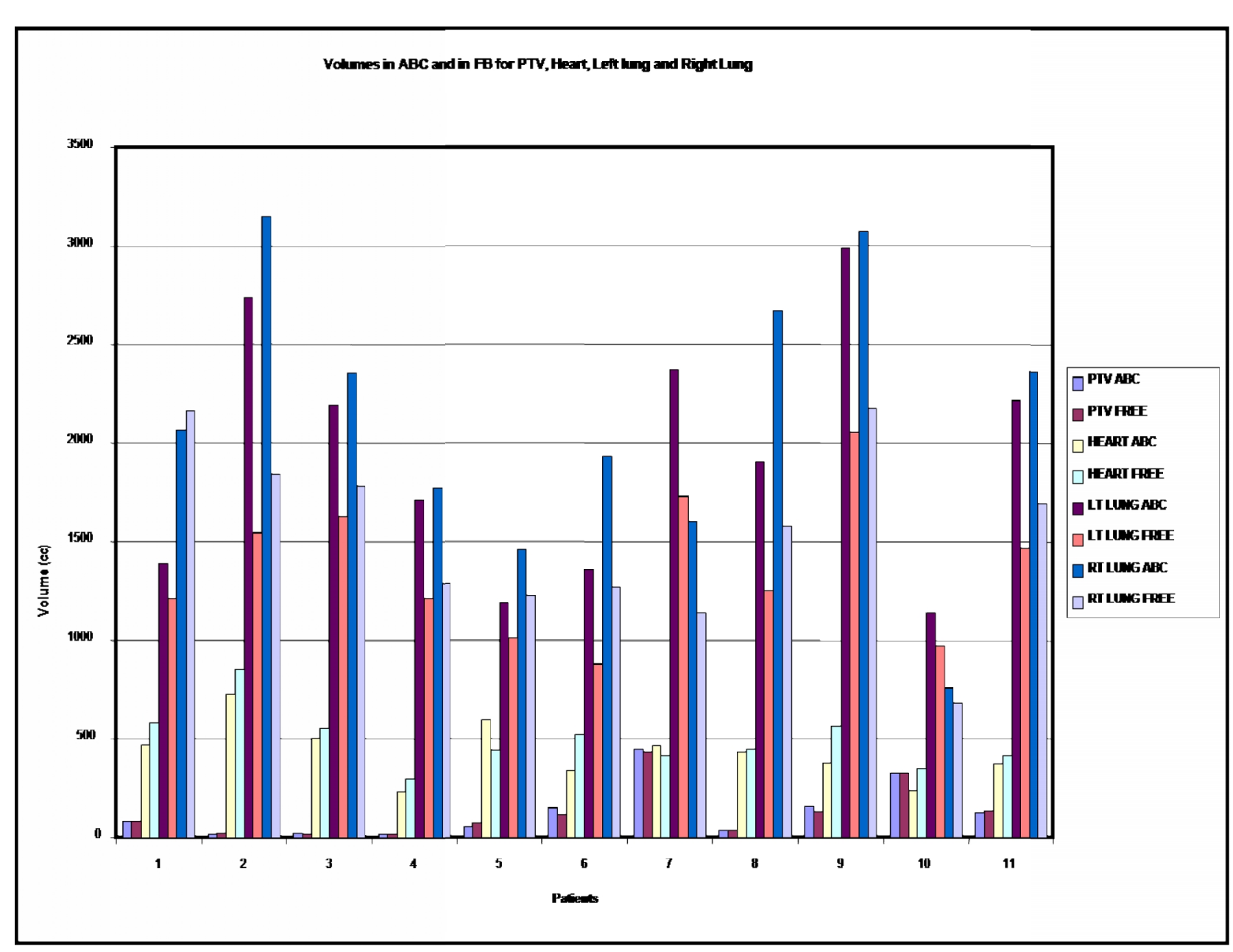

The average volumes of heart in 11 patients decreased to $-18.83 \%$ in $\mathrm{ABC}$ compared to free breathing with the range of difference is from $-49.46 \%$ to $25.31 \%(p<0.4191)$. The maximum and minimum differences were observed $-54.51 \%$ $(-183.51 \mathrm{cc})$ and $-2.9 \%(-12.73 \mathrm{cc})$ respectively. Volume comparisons for heart were illustrated in Figure 2. The range of mean dose difference in 11 patients observed was -88 cGy to $66 \mathrm{cGy}(p<0.0410)$ between $\mathrm{ABC}$ and FB. The average of minimum, mean and maximum difference in dose to the heart are $-13.34 \mathrm{cGy}(p<0.2783),-89.2 \mathrm{cGy}(p<0.0410)$ and -385 cGy $(p<0.1366)$ respectively. The range of minimum difference was from $-129 \mathrm{cGy}$ to $2 \mathrm{cGy}$ and the range of maximum difference is from -1847 cGy to 1124 cGy. The minimum, maximum and mean dose to the Heart in both FB and $A B C$ were shown in Table 1. Amount of heart receiving more than 30 Gy $\left(\mathrm{V}_{30}\right)$ were analyzed in both $\mathrm{ABC}$ and $\mathrm{FB}$ techniques and shown in Figure 3 . The mean difference of heart volume receiving more than $35 \mathrm{~Gy}$ of the dose are from 1.68 cc to -16.53 cc $(p<0.2765)$.

FIG. 2: Comparison of volumes in ABC with FB for PTV, heart, left lung, right lung. 
TABLE 1: Minimum, maximum and mean dose (cGy) to the PTV and critical structures in FB and ABC for 11 Patients.

\begin{tabular}{|c|c|c|c|c|c|c|c|c|c|c|c|c|c|c|c|c|c|c|c|c|c|c|c|c|c|c|}
\hline \multirow{3}{*}{$\#$} & \multirow{2}{*}{\multicolumn{3}{|c|}{ PTV ABC }} & \multirow{2}{*}{\multicolumn{3}{|c|}{ PTV Free }} & \multirow{2}{*}{\multicolumn{3}{|c|}{ Heart ABC }} & \multirow{2}{*}{\multicolumn{3}{|c|}{ Heart free }} & \multirow{2}{*}{\multicolumn{3}{|c|}{ RT Lung ABC }} & \multirow{2}{*}{\multicolumn{3}{|c|}{ RT Lung free }} & \multirow{2}{*}{\multicolumn{3}{|c|}{ LT Lung ABC }} & \multirow{2}{*}{\multicolumn{3}{|c|}{ LT Lung FB }} & $\begin{array}{c}\text { Spinal } \\
\text { cord ABC }\end{array}$ & $\begin{array}{l}\text { Spinal } \\
\text { cord FB }\end{array}$ \\
\hline & & & & & & & & & & & & & & & & & & & & & & & & & \multicolumn{2}{|c|}{ Dose (cGy) } \\
\hline & $\min$ & $\max$ & mean & $\min$ & $\max$ & mean & $\min$ & $\max$ & mean & $\min$ & $\max$ & mean & $\min$ & $\max$ & mean & $\min$ & $\max$ & mean & $\min$ & $\max$ & mean & $\min$ & $\max$ & mean & $\max$ & $\max$ \\
\hline 2 & 3336 & 3708 & 3595 & 3384 & 3597 & 3598 & 0 & 0 & 0 & 0 & 19.6 & 0 & 0 & 5160 & 250.6 & 0 & 5236 & 347.2 & 0 & 1120 & 82.6 & 0 & 1048 & 119 & 1493 & 1513 \\
\hline 3 & 4623 & 5206 & 5030 & 4774 & 5201 & 5035 & 0 & 667 & 25 & 0 & 1333 & 113 & 0 & 5027 & 267 & 0 & 5083 & 366 & 0 & 969 & 94 & 0 & 979 & 108 & 1435 & 1450 \\
\hline 4 & 4553 & 5183 & 5061 & 4565 & 5165 & 5029 & 5 & 850 & 157 & 3 & 714 & 91 & 0 & 5127 & 556 & 0 & 5161 & 671 & 0 & 633 & 74 & 0 & 511 & 65 & 332 & 564 \\
\hline 5 & 4067 & 5445 & 4981 & 4198 & 5329 & 4937 & 28 & 4130 & 772 & 32 & 2834 & 703 & 5 & 5312 & 1262 & 1 & 5322 & 1241 & 1 & 1593 & 261 & 1 & 1624 & 285 & 2867 & 2769 \\
\hline 7 & 1943 & 5749 & 5004 & 3945 & 6029 & 5023 & 214 & 4638 & 1347 & 343 & 5002 & 1420 & 15 & 5362 & 1293 & 25 & 6029 & 1893 & 16 & 3839 & 745 & 25 & 4646 & 787 & 3284 & 4723 \\
\hline 8 & 3994 & 5323 & 4933 & 4467 & 5198 & 5047 & 0 & 3 & 0 & $\begin{array}{l}0 \\
0\end{array}$ & 19 & 2 & 0 & 5091 & 314 & 0 & 5236 & 544 & 0 & 2043 & 172 & 0 & 2141 & 263 & 3023 & 4615 \\
\hline 9 & 3391 & 5254 & 5015 & 4469 & 5342 & 5019 & 0 & 62 & 14 & 0 & 83 & 20 & 0 & 2421 & 268 & 0 & 2896 & 210 & 0 & 5357 & 740 & 0 & 5404 & 1223 & 2092 & 1892 \\
\hline 10 & 4391 & 5262 & 5037 & 4346 & 5230 & 5044 & 37 & 2843 & 262 & 39 & 4690 & 522 & 14 & 5253 & 1984 & 23 & 5239 & 2908 & 4 & 3875 & 509 & 11 & 4718 & 574 & 4205 & 4859 \\
\hline 11 & 3437 & 4210 & 4024 & 3080 & 4197 & 4037 & 10 & 4790 & 253 & 24 & 5112 & 586.3 & 0 & 4976 & 906.3 & 5 & 5248 & 1807 & 0 & 1743 & 293 & 5 & 1867 & 416 & 1201 & 1295 \\
\hline
\end{tabular}

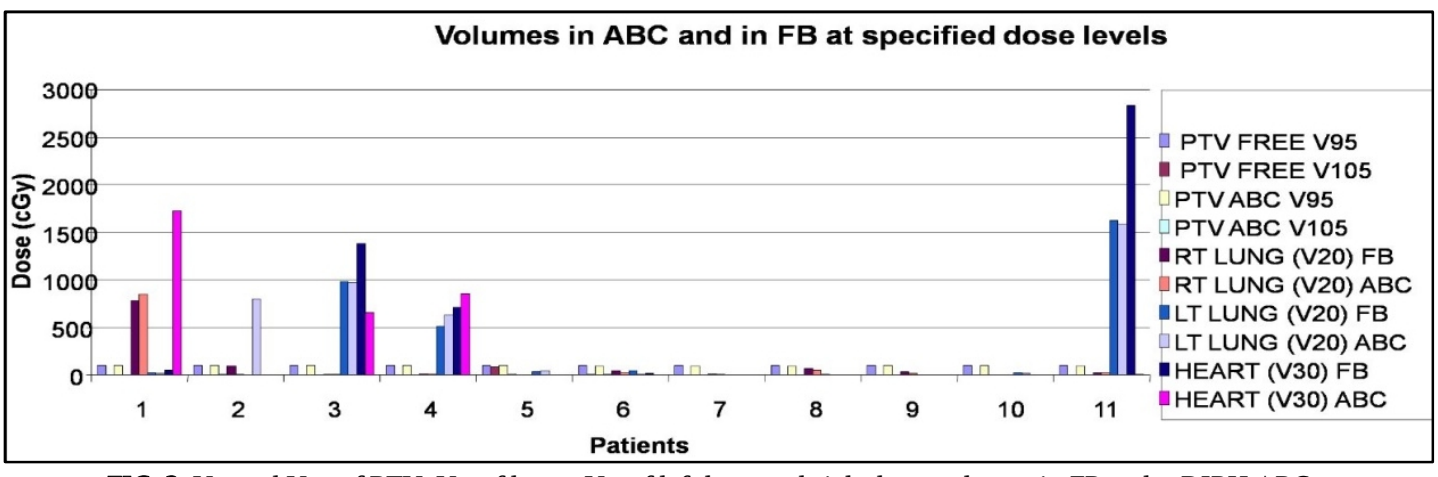

FIG. 3: $\mathrm{V}_{95}$ and $\mathrm{V}_{105}$ of $\mathrm{PTV}, \mathrm{V}_{30}$ of heart, $\mathrm{V}_{20}$ of left lung and right lung volumes in FB and mDIBH $\mathrm{ABC}$. 


\section{Left lung}

The average volumes of Left lung (after deducting the PTV volume in that particular lung ) in 11 patients increased to $27.5 \%$ in $\mathrm{ABC}$ compared to free breathing with the range of difference is from $12.66 \%$ to $43.44 \%$ ( $p<0.000168$ ). Maximum difference and minimum differences were $43.44 \%$ ( $1190 \mathrm{cc}$ ) and $12.66 \%(176 \mathrm{cc})$, respectively. The comparison of left lung volume is shown in Figure 2.

The range of mean dose difference to the left lung in 11 patients were $-483 \mathrm{cGy}$ to $9 \mathrm{cGy}(p<0.1824)$ with ABC technique when compared with free breathing IMRT. The average of minimum, mean and maximum difference in dose to the left lung are $-2.8 \mathrm{cGy}(p<0.0455),-94.9 \mathrm{cGy}(p<0.1824)$ and $-135.6 \mathrm{cGy}(p<0.4266)$ respectively. The range of minimum difference was from $-10 \mathrm{cGy}$ to $0 \mathrm{cGy}$, and the range of maximum difference was from -843 cGy to $480 \mathrm{cGy}$. The minimum, maximum and mean dose to the left lung in both FB and ABC are shown in Table 1. The $V_{20}$ of left lung was analyzed in both $\mathrm{ABC}$ and $\mathrm{FB}$ techniques and shown in Figure 3 . The mean difference of left lung volume receiving more than $20 \mathrm{~Gy}$ of the dose were from $-6.5 \%$ to $-92 \%(p<$ 0.0927).

\section{Right Lung}

The average volumes of right lung (after deducting the PTV volume in that particular lung ) in 11 patients increased to $25.85 \%$ (594.5 cc) in ABC compared to free breathing with the range of difference is from $41.33 \%$ cc to $4.62 \%$ ( $p<$ $0.001039)$. The maximum and minimum differences were $41.33 \%$ (1301 cc) and 4.62\% (100.29 cc), respectively. The comparison of right lung volume in $\mathrm{FB}$ and $\mathrm{ABC}$ is shown in Figure 2.

The range of mean difference in dose to the right lung in 11 patients were $-924 \mathrm{cGy}$ to $58 \mathrm{cGy}(p<0.0368)$ with $\mathrm{ABC}$ technique when compared with free breathing IMRT. The average of minimum, mean and maximum difference in dose to the right lung are $(-1.8 \mathrm{cGy})(p<0.1918),-264.6 \mathrm{cGy}(p<$ $0.0368)$ and $-8843 \mathrm{cGy}(p<0.3519)$, respectively. The range of minimum difference was from -10 cGy to 0 cGy and the range of maximum difference was from -667 cGy to 680 cGy. The minimum, maximum and mean dose to the right lung in both FB and $A B C$ are shown in Table 1. The $V_{20}$ of right lung was analyzed in both $A B C$ and $F B$ techniques and shown in Figure 3 . The mean difference of right lung volume receiving more than $20 \mathrm{~Gy}$ of the dose was from $1 \%$ to $-98 \%(p<$ 0.24556).

\section{Spinal cord}

The range of maximum dose difference to the spinal cord in 11 patients were -1592 cGy to $190 \mathrm{cGy}$ with $\mathrm{ABC}$ technique when compared with free breathing IMRT. The average of maximum difference in dose to the spinal cord was -382.18 cGy $(p<0.1366)$ as shown in Table 1 . The $\mathrm{V}_{45}$ of spinal cord was analyzed in both $\mathrm{ABC}$ and FB Techniques. The mean difference of spinal cord volume receiving more than $45 \mathrm{~Gy}$ was from $0.2 \mathrm{cc}$ to $0.59 \mathrm{cc}(p<0.1394)$, and out of 11 cases only one patient received more than $5 \mathrm{cc}$ of spinal cord.

\section{MUs and segments comparison}

The number of MUs was less with $A B C$ treatment compared with FB in all 11 lung patients (Figure 4). The mean $\%$ of variation was $-22.9 \%$, the maximum $\%$ of variation was $-66.4 \%$, and the minimum \% of variation was $0.497 \%$, whereas the difference in mean \% variation was $12.46 \%$ ( $p<$ 0.0589 ). Out of eleven patients only one patient result shown negative with more number of monitor units compared to FB. It was found that more number of segments were required in $\mathrm{ABC}$ compared to $\mathrm{FB}$ (Figure 5). The minimum, maximum, and mean $\%$ variation were $0 \%, 16.39 \%$ and $58.22 \%$, respectively. The mean difference in $\%$ variation was $23.10 \%$ ( $p<$ 0.0186).

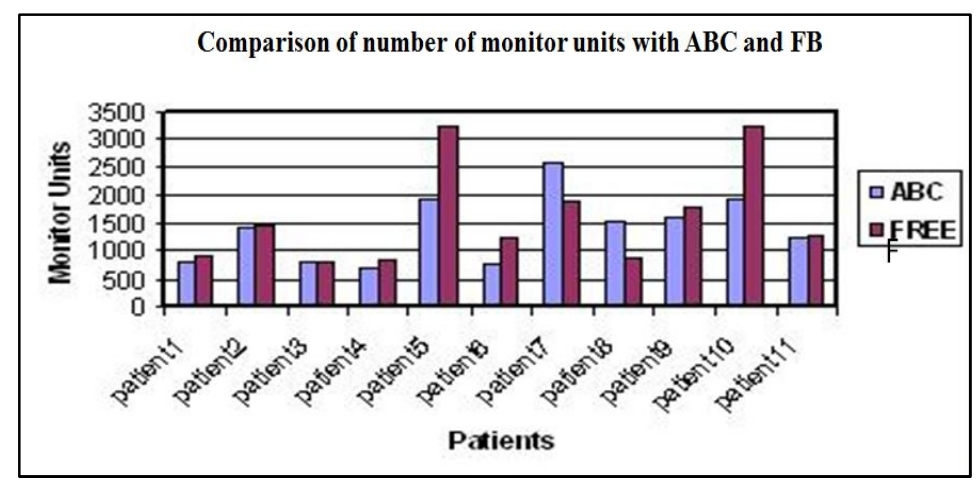

FIG. 4: Comparison of number of monitor units (MUs) in ABC and FB.

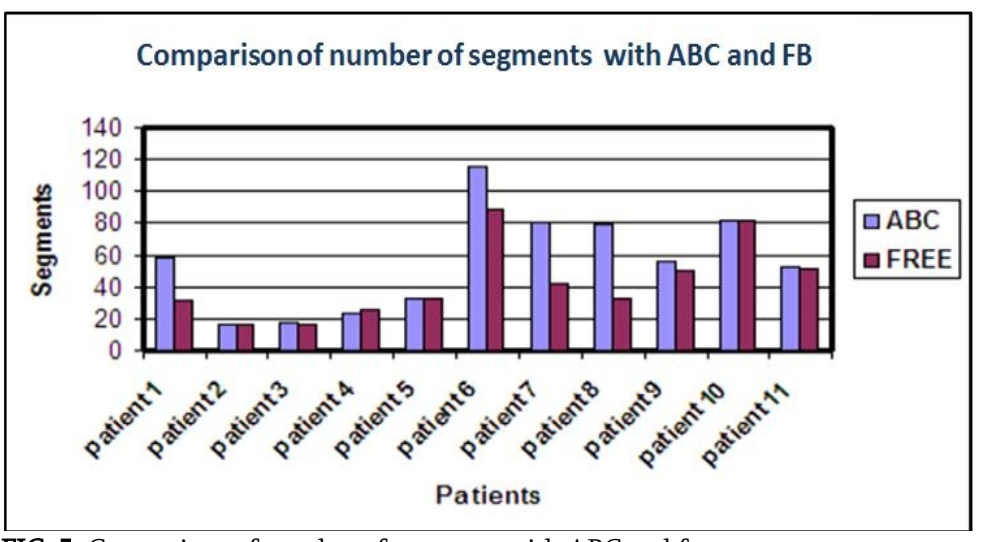

FIG. 5: Comparison of number of segments with $A B C$ and free breath.

\section{Discussion}

The present study explores improvement achieved by the mDIBH if it is based on $\mathrm{ABC}$ with $\mathrm{mDIBH}$ rather than free breathing CT scan using identical treatment plan parameters. It is acknowledged that the free breathing scan represents 
only one arbitrarily selected state of the patient. This result in the inconsistency of data (for example, in two patients the heart dose decreases in the free breathing based plan).

The mDIBH significantly reduced the heart, lung, and spinal cord doses compared with FB, while maintaining excellent coverage of the PTV. However, the advantages of $\mathrm{mDIBH}$ compared with the FB technique depend on few factors such as patient breathing capacity, anatomy, dose rate of the machine, etc.

Patient selection is also one of the important factors. An old person who cannot perform breath control and a young child who cannot understand the procedure are not ideal patients for the treatment. In reality the free breathing scans cannot be taken at a predefined phase. In fact, scans at some particular phase are taken even during the free breathing. If all the free breathing scans are done in inspiration in the same phase where $A B C$ has been done, we may not see the much difference between both approaches. Due to this reason, we have taken 11 patients for this study. The randomness of the free breathing scan needs to be analyzed. But with ABC, we are certain about the phase in which the treatment will be delivered.

With high dose rate machines the treatment time will come down, which is very much useful to perform ABC. Anatomy is also very important factor. As each patient is having unique structure the statistical data that was obtained in few cases were insignificant. In few cases where $\mathrm{mDIBH}$ have no significant effect and are with contradict results, those patients with other threshold or phase of the treatment should be taken for analysis to get the optimal phase of the treatment. Because of increased space between the other organs at risk and the PTV during particular phase of the breathing cycle, the target coverage and protection to OAR's can be achieved. The use of $\mathrm{mDIBH}$ with $\mathrm{ABC}$ produced no significant difference in PTV volume for $\left(\mathrm{V}_{95}\right)$ and $\left(\mathrm{V}_{105}\right)$. ABC reduced the number of MUs and increased the segments.

In the present study, the average mean heart dose for 11 patients decreased to $76.3 \%$. This is a significant result. But in two cases, it showed the maximum dose in $A B C$ is more compared to FB. This necessitates further study on gating. During gating, treatment will be delivered in some particular phase only. There is a need to check the suitability of the phase to the ideal treatment. The best phase of the treatment is the phase where critical structures should be as far away from the target as possible in the beams eye view of the beam in the treatment planning. Because of this at least few phases should be thoroughly analyzed instead of going for treatment with gating. Figure 6 shows the different phases on full cycle of breathing.

As demonstrated in Figure 3, the impact of mDIBH on heart calculations shows good results as observed with heart $\mathrm{V}_{30}$ values. However, because the dose reduction with $\mathrm{mDIBH}$ occurs primarily in the anterior part of the heart where the coronary vessels are positioned, significantly greater benefits in reducing long-term ischemic disease might be expected. But, we can see the discrepancy for two patients who are getting more volume $\left(\mathrm{V}_{30}\right)$ in $\mathrm{ABC}$ compared with $\mathrm{FB}$. This is the interesting area in the present paper, and we need more attention to avoid such negative results that may happen with any gating method.

Margin reduction can be achieved by taking into account the breathing motion and setup errors. The margin will vary for each and individual patient. This information is very useful for adaptive radiation therapy. ${ }^{15-16}$ Drastic reduction in lung dose was observed with mDIBH in our study by considering a cutoff value of lung $\mathrm{V}_{20}$ (both lungs) for all 11 patients. In this paper, if one considers the individual case, the negative sign shown implies that some patients are getting more minimum dose and maximum dose compared with FB. The aim of this paper is to highlight these figures. The same types of results were already discussed in case of heart, left lung, and spinal cord also. The results were dependent on anatomy. But there is no consistent data for all the patients as discussed above. Furthermore, the treatment with $A B C$ needs decreased amount of MUs necessary to deliver a treatment fraction. The decrease in treatment time helps to minimize the patient motion during treatment.

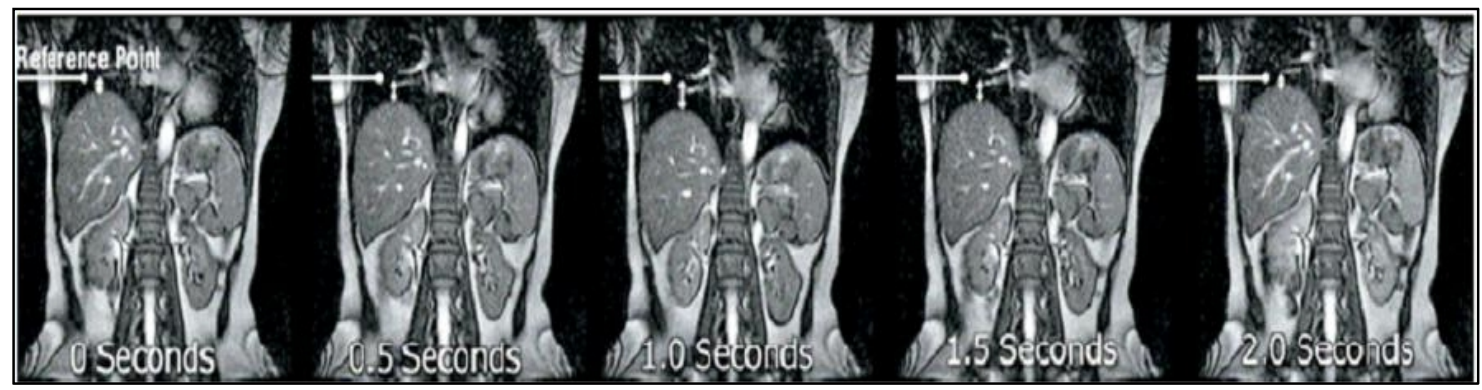

FIG. 6: Different phases of the breathing cycle. (Courtesy by Varian) 
The solution for all these problems like inconsistency and increased dose to the normal structures can be solved by

- $\quad$ performing scan, plan and treatment with optimal patient positioning and optimal phase of $\mathrm{mDIBH}$,

- $\quad$ accurate contouring with margins including intra fractional shifts during $A B C$,

- considering the variation in patient shape during treatment,

- fusion with MRI, PET-CT scans with the images that were obtained with $A B C$ in the same phase where planning CT was taken, and

- moving critical structures as much away from target as possible in the BEV of the beams with $\mathrm{mDIBH}$ or in any phase in gating technique.

\section{Conclusion}

Bases on the results of 11 patients presented in this study, we conclude that radiotherapy of lung cancer with $\mathrm{ABC}$ has the potential to reduce the mean dose to heart, lung, and spinal cord compared with free breathing in most of the cases. ABC technique needs fewer MUs, thus decreasing the treatment time which further provides patient comfort. $A B C$ technique can immobilize the tumor position but there is no guarantee that it can spare critical structures for all patients compared with FB. This is due to the possibility of critical structures coming into the field for longer time in $\mathrm{ABC}$ technique, and patient's anatomy, which influences the results.

\section{Conflict of interest}

The authors declare that they have no conflicts of interest. The authors alone are responsible for the content and writing of the paper.

\section{References}

1. Tyldesley S, Boyd C, Schulze K, et al. Estimating the need for radiotherapy for lung cancer: an evidence-based, epidemiologic approach. Int J Radiat Oncol Biol Phys 2001; 49:973-85.

2. Barbera L, Zhang-Salomons J, Huang J, et al. Defining the need for radiotherapy for lung cancer in the general population: a criterion-based, benchmarking approach. Med Care 2003; 41:1074-85.

3. Truong MT, Erasmus JJ, Gladish GW, et al. Anatomy of pericardial recesses on multidetector CT: implications for oncologic imaging. AJR Am J Roentgenol 2003; 181:1109-13.

4. Ozmen CA, Akpinar MG, Akay HO, et al. Evaluation of pericardial sinuses and recesses with 2-, 4-, 16-, and 64-row multidetector CT. Radiol Med 2010;115:1038-46.

5. AAPM Report 91. The management of respiratory motion in radiation oncology. American Association of Physicists in Medicine 2006. Available from http://www.aapm.org/pubs/reports/RPT_91.pdf

6. Muralidhar KR, Murthy PN, Mahadev DS, et al. Magnitude of shift of tumor position as a function of moderated deep inspiration breath-hold: An analysis of pooled data of lung patients with active breath control in image-guided radiotherapy. JMed Phys 2008; 33:147-53.

7. Dawson LA, Brock KK, Kazanjian S, et al. The reproducibility of organ position using active breathing control (ABC) during liver radiotherapy. Int J Radiat Oncol Biol Phys 2001; 51:1410-21.

8. Case RB, Sonke JJ, Moseley DJ, et al. Inter- and intrafraction variability in liver position in non-breath-hold stereotactic body radiotherapy. Int $J$ Radiat Oncol Biol Phys 2009; 75:302-8.

9. Hurkmans CW, Remeijer P, Lebesque JV, Mijnheer BJ. Set-up verification using portal imaging; review of current clinical practice. Radiother Oncol 2001; 58:105-20.

10. Pehlivan B, Pichenot C, Castaing M, et al. Interfractional set-up errors evaluation by daily electronic portal imaging of IMRT in head and neck cancer patients. Acta Oncol 2009; 48:440-5.

11. Amankwaa-Frempong E, Vernimmen F, Blay S, Ezhilalan R. Irradiation of lung and esophagus tumors: A comparison of dose distributions calculated by anisotropic analytical algorithm and pencil beam convolution algorithm, a retrospective dosimetric study. Int J Cancer Ther Oncol 2014; 2:020210.

12. Rana S, Pokharel S, Zheng Y, et al. Treatment planning study comparing proton therapy, RapidArc and intensity modulated radiation therapy for a synchronous bilateral lung cancer case. Int J Cancer Ther Oncol 2014; 2:020216.

13. Chaikh A, Giraud J, Balosso J. A method to quantify and assess the dosimetric and clinical impact resulting from the heterogeneity correction in radiotherapy for lung cancer. Int J Cancer Ther Oncol 2014; 2:020110.

14. Middelton MR. Data analysis using Microsoft Excel. Updated for office 97 \& 98. Pacific Grove, CA: Duxburry Thomson Learning, Brooks/Cole; 2000; 133-136.

15. Martinez AA, Yan D, Lockman D, et al. Improvement in dose escalation using the process of adaptive radiotherapy combined with three-dimensional conformal or intensity-modulated beams for prostate cancer. Int J Radiat Oncol Biol Phys 2001; 50:1226-34.

16. Ghilezan M, Yan D, Liang J, et al. Online image-guided intensity-modulated radiotherapy for prostate cancer: How much improvement can we expect? A theoretical assessment of clinical benefits and potential dose escalation by improving precision and accuracy of radiation delivery. Int J Radiat Oncol Biol Phys 2004; 60:1602-10. 